\title{
Comparison between RF and electrical signals from the partial discharge activity of twisted pair cables at reduced pressures
}

\author{
M. J. Given, R. Mason, M. Judd, P. McGlone, I. V. Timoshkin, and M. P. Wilson \\ Department of Electrical Engineering \\ University of Strathclyde \\ Glasgow G1 1XW UK
}

\begin{abstract}
A pressure-controlled test facility has been set up that allows the PD behaviour of polymer insulated twisted pair samples exposed to $50 \mathrm{~Hz} \mathrm{AC}$ voltages in the range of 0 to $10 \mathrm{kV}$ to be characterised. Resulting PD activity is quantified using the methods defined in IEC standard 60270 and by using a simple monopole antenna to detect the RF signals excited inside the pressure vessel by the discharges. This paper gives the results of preliminary tests performed on samples of wire insulated with Ethylenetetraflourethylene, Silicon Rubber and Polyvinylchloride in the pressure range between $10^{3}$ and $10^{5} \mathrm{~Pa}$ in atmospheric air. The dependence of PD inception voltage on the environmental pressure is reported. Changes in the behaviour of the PD activity; the correlations between the RF and electrical measurements and the frequency components of the RF signals as the applied voltage and pressure are varied are characterised and discussed.
\end{abstract}

\section{INTRODUCTION}

The reliable performance of electrical wiring systems plays an important role in aerospace power systems. Modern aircraft have extensive wiring systems and these are exposed mechanical, thermal, electrical and chemical stressing in the course of normal operation. In addition these systems are exposed to large variations of pressure [1]. The ageing induced by these stresses is considered to be a precursor to arcing between the conductors in the wiring system [2,3].

The behavior of partial discharge (p.d.) activity is strongly affected by the environmental pressure that the insulation system experiences. Work has been performed under low pressure conditions to investigate the p.d. behavior of twisted pair samples and dielectric barrier discharge geometries [4] using methods based on the IEC 60270 standard.

This paper reports on the development of a test system which will allow the partial discharge behavior of twisted pair samples at reduced pressures to be monitored using both the IEC methodology and RF detection techniques.

\section{Experimental System}

\section{A. Test Chamber and Sample Holder.}

An aluminium high vacuum chamber was used for this project with customized high voltage connectors.

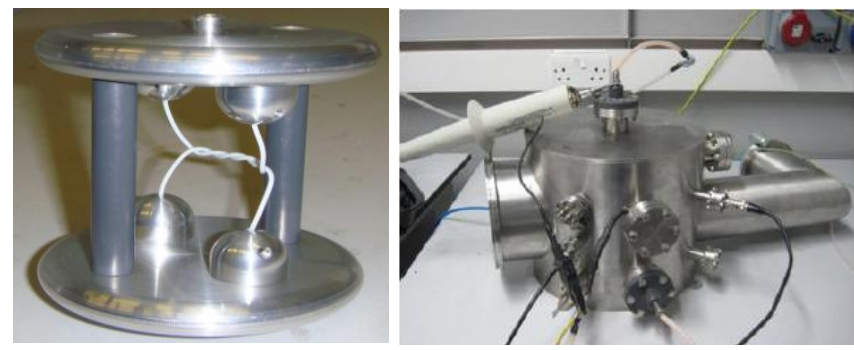

Fig. 1 Sample holder and test chamber.

The twisted pair samples were held in a custom built jig (Fig. 1). The IEC measurement system which is described below was held within the vacuum chamber. In the experimental tests that were performed a rotary pump was used to evacuate the chamber achieving a minimum pressure of $-85 \mathrm{kPa}$ gauge.

\section{B. Samples Used}

Three types of wire samples were used in these initial tests: An ETFE insulated wire with an outer diameter of $1.3 \mathrm{~mm}$ and an insulation thickness of $0.25 \mathrm{~mm}$; A Silicon Rubber insulated wire with an outer diameter of $1.85 \mathrm{~mm}$ and an insulation thickness of $0.7 \mathrm{~mm}$ and a PVC insulated wire with an outer diameter of $2.75 \mathrm{~mm}$ and an insulation thickness of $1 \mathrm{~mm}$.

Lengths of the wire were formed into twisted pairs with 6 twists in a $5 \mathrm{~cm}$ length. A force of $40 \mathrm{~N}$ was applied during the twisting process.

\section{IEC 60270 and RF Measurement System}

The IEC measurement system consisted of a $100 \mathrm{pF}$ vacuum capacitor in series with a $50 \Omega$ viewing resistor. This combination was placed in parallel with the test sample. Fig. 2. The viewing resistor was connected to a $50 \Omega$ BNC vacuum lead-through. The RF antenna consisted of a $10 \mathrm{~cm}$ length of copper wire connected to a second BNC vacuum lead-through. The output of the two sensors were then connected through $50 \Omega$ coaxial cables to an oscilloscope an inline filter was included on the RF connection to remove the $50 \mathrm{~Hz}$ component of the signal. The system was stressed using a modified Foster oil test transformer capable of providing a maximum output of $25 \mathrm{kV}$ in the configuration used. 


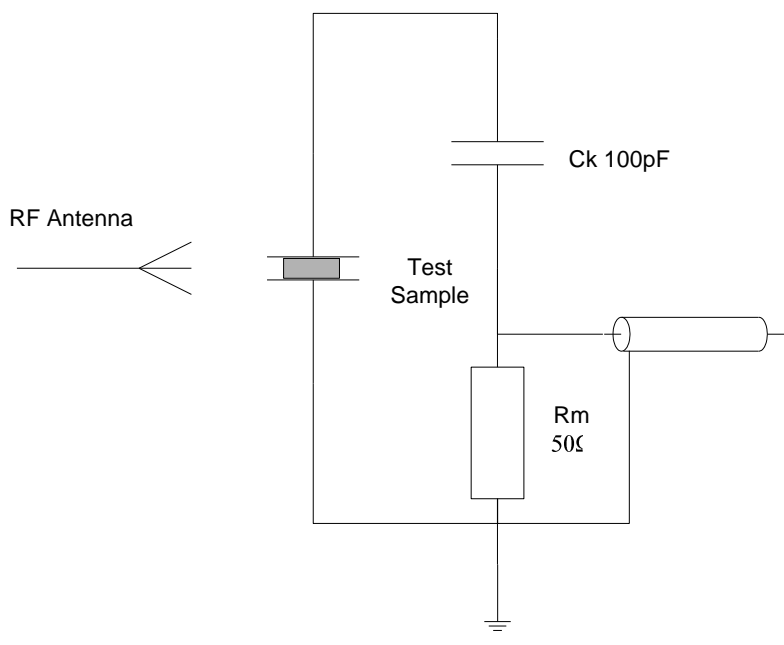

Fig. 2 Schematic of Measurement System

In initial tests, the basic IEC system used was shown to be capable of detecting partial discharges and signals of the order of $10 \mathrm{~V}$ were observed from the system Fig. 3. The output of the antenna for the RF measurements was of the order of $200 \mathrm{mV}$ and could be directly observed using the oscilloscope. Fig. 3. To ensure that the p.d. signals were originating from the twisted pair and not from the sample holder or measurement system, the twisted pair sample was replaced with two loops of wire which were not in contact. No discharge activity was observed in this case for the range of voltages used.

\section{EXPERIMENTAL RESULTS}

\section{A. Behaviour at Atmospheric Pressure}

An example of the measured output of the IEC and RF systems for a partial discharge event are shown in Fig.3.

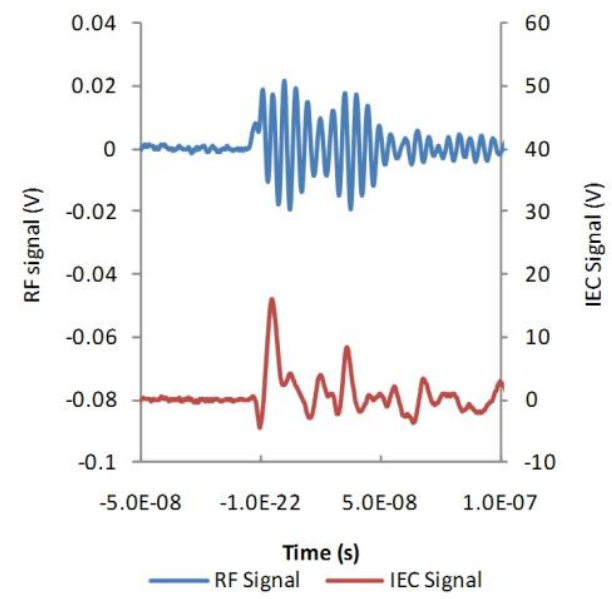

Fig 3 Output of IEC and RF detection systems to a p.d. event in ETFE sample.

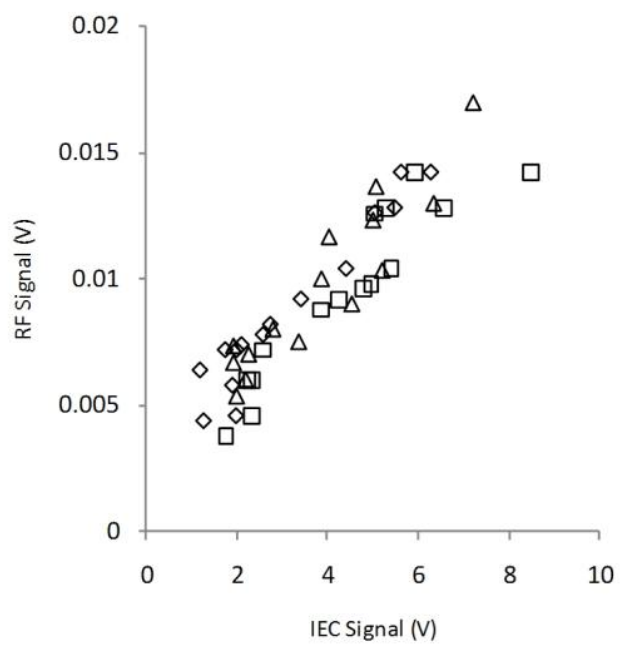

Fig. 4 Comparison of signal peak data for IEC and RF detection systems. $\triangle$ ETFE, $\square$ SIR, $\diamond$ PVC

Data was collected for the peak values for both signals for the three sample types over a range of voltages at atmospheric pressure. The results are shown in Fig. 4. It can be seen that there is a reasonable correlation between the peak signals produced by the two different monitoring systems.

Spectral analysis was performed on the output waveforms obtained from the measurement systems. Fig. 5a shows a frequency spectrum for a ETFE sample at atmospheric pressure at an applied voltage of $2.5 \mathrm{kV}$. It can be seen that there is a major peak at $40 \mathrm{MHz}$ and smaller peaks occurring at $90,120,150$ and $210 \mathrm{MHz}$. Fig. 5b shows the corresponding spectrum for the RF probe data. In this spectrum there is a clear peak at $210 \mathrm{MHz}$ with a suggestion of a secondary peak at $225 \mathrm{MHz}$. No significant changes in the spectral content were observed as the voltage applied to the ETFE samples was changed

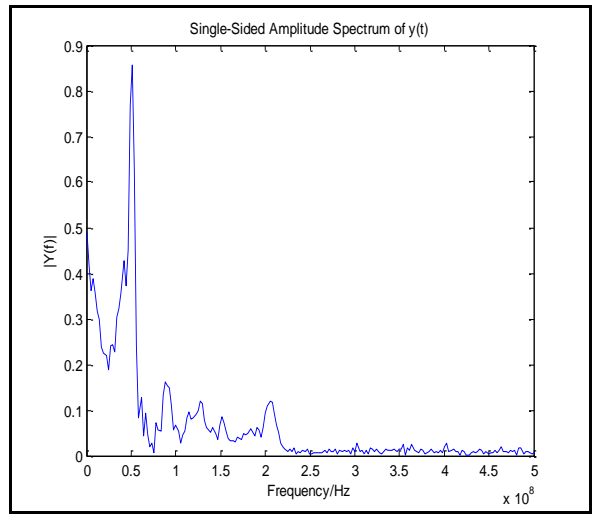

Fig. 5a spectrum from IEC measurements for EDFE at $2.5 \mathrm{kV}$ 


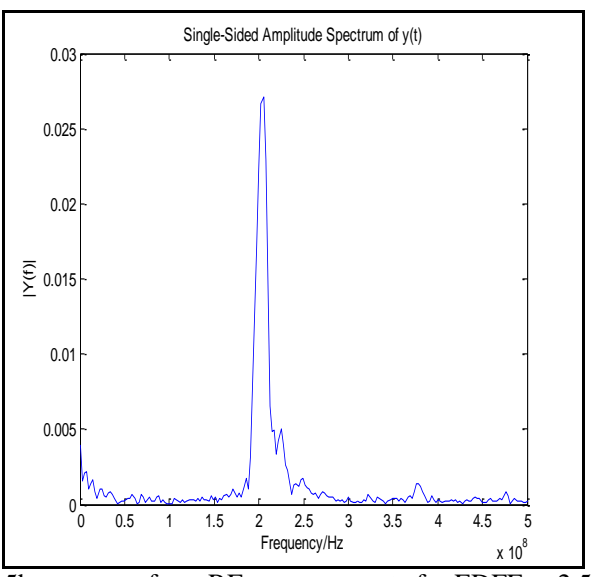

Fig. $5 \mathrm{~b}$ spectrum from RF measurements for EDFE at $2.5 \mathrm{kV}$

Fig. 6a shows the frequency spectrum for the signal from the IEC probe for a p.d. event at in a SIR sample at atmospheric pressure at a voltage of $3.2 \mathrm{kV}$. Like the spectra for ETFE the spectrum contains a major peak at $40 \mathrm{MHz}$. However, above this frequency there are differences in the spectrum with an additional peak appearing at $70 \mathrm{MHz}$ and the peak at $210 \mathrm{MHz}$ being suppressed.

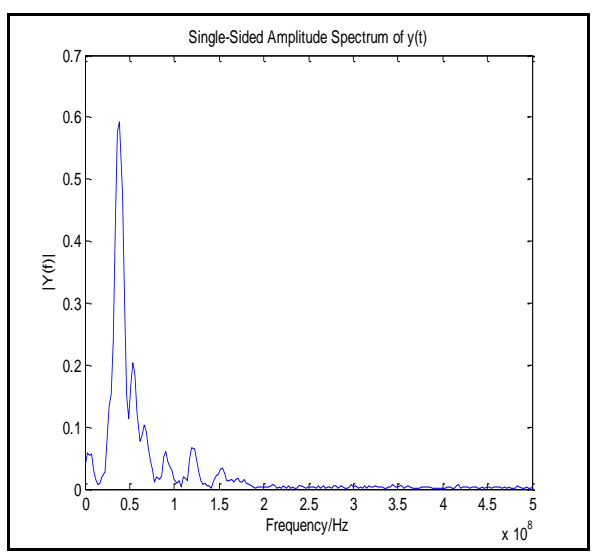

Fig. 6a spectrum from IEC measurements for SIR at $3.2 \mathrm{kV}$

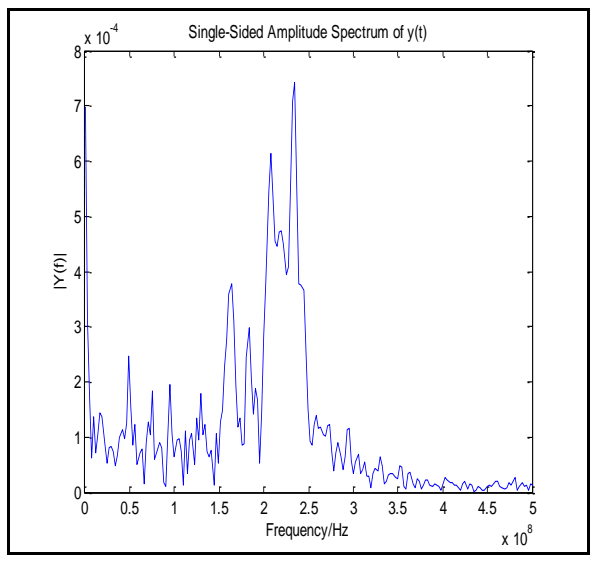

Fig. $6 \mathrm{~b}$ spectrum from RF measurements for SIR at $3.2 \mathrm{kV}$
The corresponding RF spectrum, Fig. 6b, maintains the strong double peak structure but the intensity of the peaks below $200 \mathrm{MHz}$ is strongly reduced. There is also a clear change in the frequency content of the UHF signal for SIR compared to ETFE under these conditions with a clear double peak at frequencies of 210 and $240 \mathrm{MHz}$. The most obvious difference is that rather than seeing a single peak with a shoulder at $210 \mathrm{MHz}$ a clear double peak at frequencies of 210 and $240 \mathrm{MHz}$. Another smaller double peak feature appears between 150 and $200 \mathrm{MHz}$ and a series of small peaks can be seen in the spectrum between 50 and $125 \mathrm{MHz}$.

At higher voltages, changes in both the IEC spectrum Fig. 6c and the RF spectrum Fig. $6 \mathrm{~d}$ are observed. At $4.0 \mathrm{kV}$ the IEC spectrum still shows a clear signal at $40 \mathrm{MHz}$ but the relative magnitude of the peaks at 70,90 and $120 \mathrm{MHz}$ has increased.

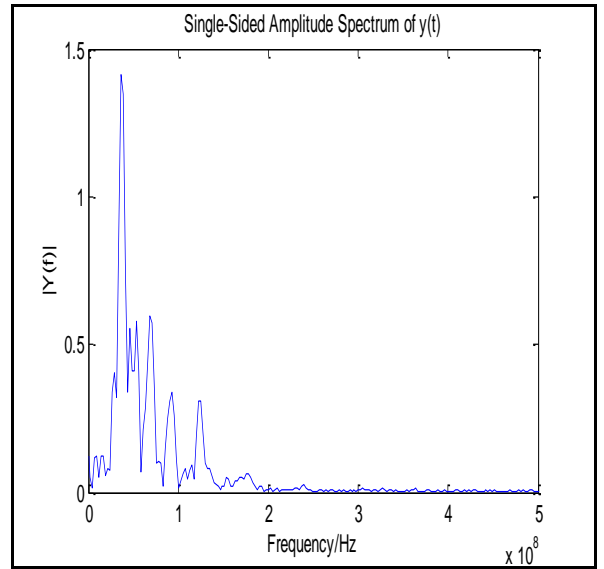

Fig. 6c spectrum from IEC measurements for SIR at $4.0 \mathrm{kV}$

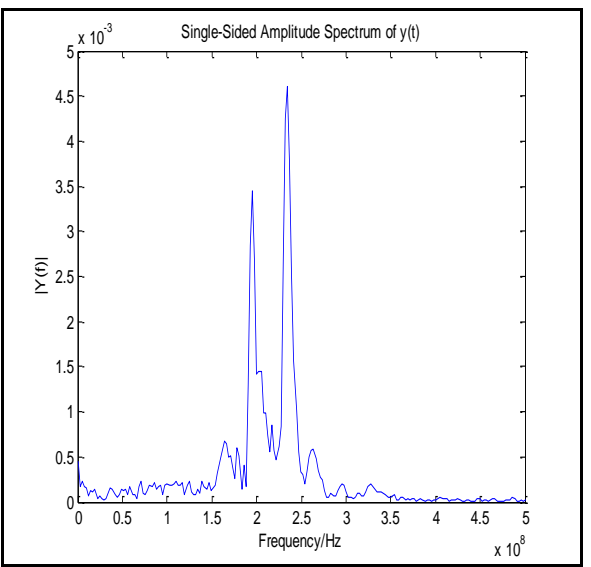

Fig. 6d spectrum from RF measurements for SIR at $4.0 \mathrm{kV}$

Fig. 7a Shows the IEC spectrum for a p.d. event in the $\mathrm{PVC}$ insulated system at a voltage of $3.75 \mathrm{kV}$. Again a peak is observed a major peak is observed at $40 \mathrm{MHz}$. As with ETFE peaks are also observed at 90, 120 and $150 \mathrm{MHz}$. However the relative amplitude of these peaks is stronger than those 


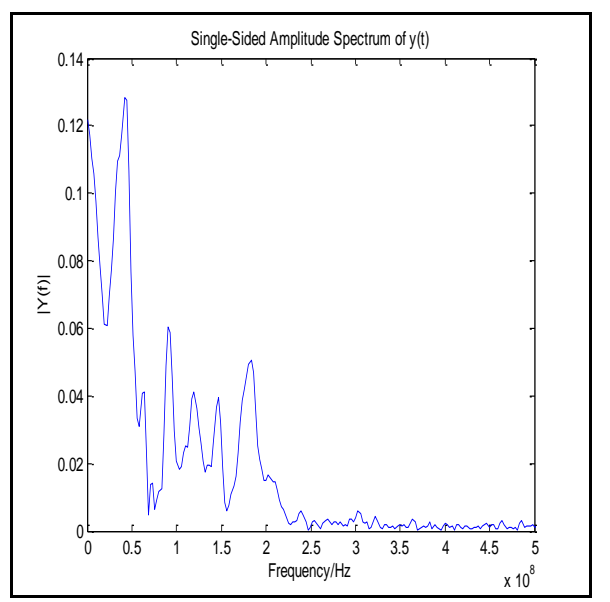

Fig. 7a spectrum from IEC measurements for PVC at $3.75 \mathrm{kV}$

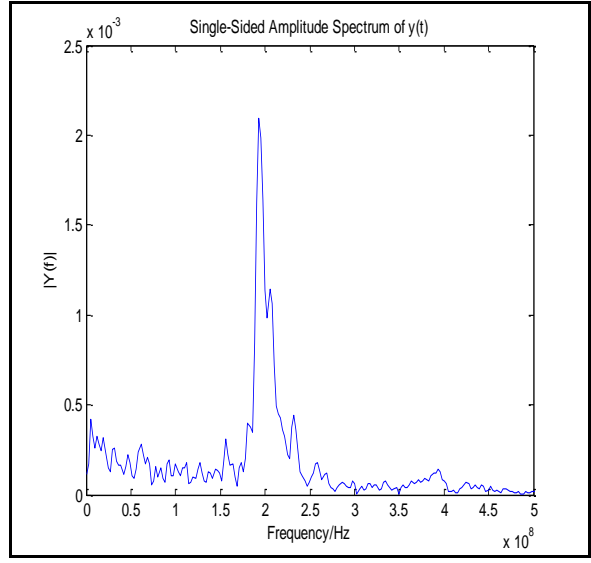

Fig. 7b spectrum from IEC measurements for PVC at $3.75 \mathrm{kV}$

observed in ETFE. The RF spectrum, Fig. 7b, shows a major peak close to $190 \mathrm{MHz}$ with suggestions of a second peak at 210 MHz. No significant changes in the spectra of the discharge events were observed for the PVC samples as the applied voltage was increased.

\section{B Behaviour at reduced pressures}

The initiation voltage for p.d activity as a function of pressure was investigated and the results obtained are illustrated in Fig. 8. As has been reported in the literature [5] the initiation voltage decreases as the pressure is reduced. The measured peak values of both the IEC and RF signals was also observed to increase as the pressure was reduced.

In certain cases when measurements were performed at reduced pressure for the SIR and PVC insulation systems, the magnitude of the output of the IEC probe lead to clipping of the recorded waveforms. This made it impossible to accurately analyse the frequency content of the spectra of these signals. Preliminary evaluation of the spectral content of the p.d. signals in ETFE show no significant changes in the IEC signals as the pressure is changed.

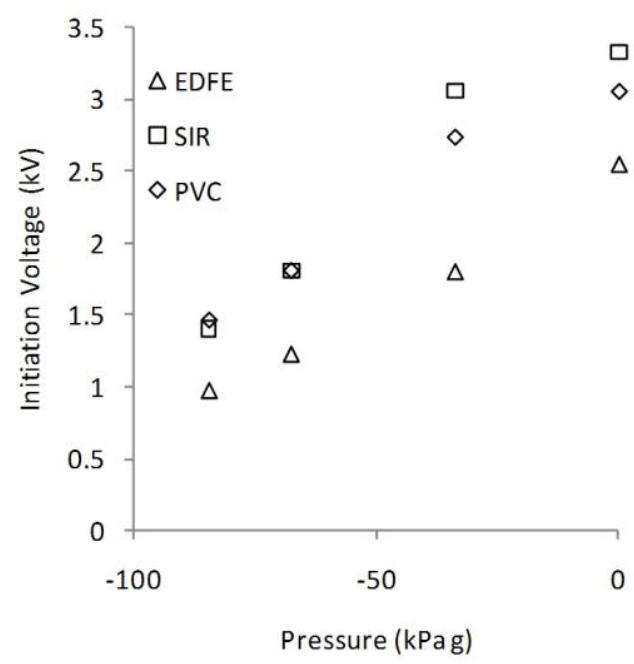

Fig. 8 Initiation voltage for p.d. activity

\section{CONCLUSIONS}

A test system has been developed that allows the partial discharge activity of twisted pair samples to be investigated over a range of pressures using both an IEC 60270 and a RF measurement system. For the three insulation systems considered partial discharge activity was observed using both detection systems and correlation was possible between the IEC and RF signals.

Examining the spectral content of both the IEC and RF signals clear differences can be observed for the three insulation systems used in this initial study observed at atmospheric pressure. As expected the behavior of both the IEC signals the partial discharge activity for the three samples was dependent on the pressure minor modifications are required to the IEC measurement system to deal with the signal magnitudes generated at low pressures to allow a detailed examination to be undertaken.

\section{ACKNOWLEDGMENT}

The results presented in this paper are come are based on work performed by Mr Feng Ding and Ms. Xiaoxin Liu as part of their MSc by Instruction and B.Eng Degree project work at the University of Strathclyde.

\section{REFERENCES}

[1] J. S. Kuzniar, G. A. Slenski, "Wire Integrity Field Survey of USAF Legacy Aircraft," Proceedings of the RTO AVT Specialists' Meeting on Life Management Techniques for Aging Air Vehicles, RTO-MP-079, 2001.

[2] Y. X. Wang, E. Y. Dong, J. Y. Cong, J. Y. Zou, D. Koenig, ”Arc Tracking Properties of the Aging Wires in Aircraft," IEEE, 1-42442386-6, June 2008

[3] F.R. Frontzek, D. Koenig, M.D. Judd, H.J. Reher, “ Fault Arc Propagation on Cables for Space Applications in Vacuum, Oxygenenriched Air and Air" IEEE Transactions on Dielectrics and Electrical Insulation, Vol 2 no 2, 1995, pp 190-7

[4] X Liu "Partial Discharge Detection and Analysis in Low Pressure Environments" Ph.D. Thesis Ohio State University USA 2006 